\title{
THE DECEMBER MEETING OF THE AMERICAN MATHEMATICAL SOCIETY AT CHICAGO.
}

The thirty-eighth regular meeting of the Chicago Section, being the seventh regular meeting of the Society at Chicago, took place at the University of Chicago on Friday, December 22. The meeting was attended by fifty-eight persons, a number only slightly below the usual total of attendance registrations. In view of the fact that the date of this meeting was very inconvenient, coming during the last days preceding Christmas, this nearly normal attendance may be looked upon as very encouraging. The following forty-two members of the Society were present:

Dr. Florence E. Allen, Professor F. Anderegg, Professor G. N. Bauer, Professor A. A. Bennett, Professor G. A. Bliss, Professor Henry Blumberg, Professor H. T. Burgess, Professor W. D. Cairns, Professor R. D. Carmichael, Mr. E. H. Clarke, Professor H. E. Cobb, Professor G. H. Cresse, Professor D. R. Curtiss, Dr. W. W. Denton, Professor L. E. Dickson, Professor L. W. Dowling, Professor Arnold Dresden, Professor Arnold Emch, Professor W. B. Ford, Professor A. S. Hathaway, Professor T. H. Hildebrandt, Mr. Glenn James, Professor A. M. Kenyon, Dr. W. W. Küstermann, Professor Kurt Laves, Professor A. C. Lunn, Professor H. W. March, Professor William Marshall, Professor E. H. Moore, Professor C. C. Morris, Professor E. J. Moulton, Professor F. R. Moulton, Dr. J. R. Musselman, Miss I. M. Schottenfels, Dr. A. R. Schweitzer, Professor J. B. Shaw, Professor C. H. Sisam, Professor H. E. Slaught, Professor E. J. Townsend, Professor E. B. Van Vleck, Professor E. J. Wilczynski, Professor J. W. A. Young.

The chairman of the Section, Professor W. B. Ford, presided at the two sessions. In the evening forty-seven members and friends dined together at the Quadrangle Club.

Plans are being made for including in the programme of the spring meeting a symposium on the subject of "The Lebesgue integral," to which an entire afternoon session is to be devoted.

The following papers were presented at this meeting:

(1) Dr. G. E. Wahlin: "The cubic equation in a $p$-adic domain." 
(2) Mr. Guenn James: “ Certain restrictions upon methods of summing divergent series."

(3) Dr. A. R. Schweitzer: "Some remarks concerning quasi-transitive functional equations."

(4) Professor H. T. Burgess: "Note on the solution of the matrix equation $X^{-1} A X=N$."

(5) Professor Henry Blumberg: "Certain general properties of functions."

(6) Professor A. S. Hathaway: "Certain residue groups."

(7) Professor E. B. VAN Vleck: "A proof of Haskins' momental theorem."

(8) Professor G. A. BuIss: "A device applicable to the problems of Lagrange and Mayer in the calculus of variations."

(9) Professor C. H. Sisam: "On the order of surfaces generated by systems of algebraic curves."

(10) Professor L. E. Dickson: "On the history of the theory of numbers (preliminary communication)."

(11) Professor F. R. Moulton: "An extended solution of differential equations."

(12) Professors G. N. Bauer and H. L. Slobin: "A system of algebraic and transcendental equations."

(13) Professor A. A. Bennetr: "Closed algebraic correspondences."

Dr. Wahlin's paper was read by title. Abstracts, numbered to correspond to the titles in the list above, follow below.

1. In this paper Dr. Wahlin makes a study of the general cubic equation for the domain of a rational prime $p$. He determines the conditions which the coefficients must satisfy in order that the cubic shall be irreducible or shall have one or three roots in $k(p)$. The result is then used and the necessary extension made for the complete determination of the factorization of $p$ in the cubic domain.

2. Mr. James considers some of the necessary restrictions upon any method of summing series by the use of convergence factors, the assumption being made that the method satisfies "the conditions of consistency." In particular he proves that the limit of the sum of the convergence factors is unity, provided the method sums at least one convergent series, whose sum is not zero, to its ordinary sum and the limit of each of 
the first $K$ factors is zero. In the second part of his paper he shows that $\lim _{x=1} \Sigma a_{n} x^{n}$ cannot exist for a certain class of oscillating series.

3. Referring to a previous* paper, Dr. Schweitzer points out that it is possible to replace, in each theorem there given, all functional equations (in the hypothesis) but one by certain rational conditions on the $M$ 's. In particular, in the cubic case, the theorem as stated by the author (l. c.) remains valid if one of the functional equations is dropped from the hypothesis.

As subcategories of categories of functional equations previously $\dagger$ defined, the author defines (1) the quasi-transitive of degree $(n+1)(n=1,2,3, \cdots)$ and order $k, 0<k \leqq n+1$, (2) the transitive of degree $(n+1)$ and order $k, 0<k \leqq n+1$. The degree is defined as the number of arguments of any one of the functions involved in the equation; the order is the degree less the number of variables eliminated. If the order equals the degree, the concepts "quasi-transitive" and "transitive" coalesce and one has as class subordinate to the resulting class the "iterative and non-eliminative" equation. Examples: $\phi\left\{f\left(t, x_{2}, x_{3}\right), f\left(x_{1}, t, x_{3}\right), f\left(x_{1}, x_{2}, t\right)\right\}$ $=f\left(x_{1}, x_{2}, x_{3}\right)$ is a transitive equation of degree three and order two; $\phi\left\{f\left(t_{1}, t_{2}, x_{3}\right), f\left(t_{1}, x_{2}, t_{2}\right), f\left(x_{1}, t_{1}, t_{2}\right)\right\}=f\left(x_{1}, x_{2}, x_{3}\right)$ is a transitive equation of degree three and order one; $f\left\{f\left(x_{1}, x_{2}, x_{3}\right)\right.$, $\left.f\left(x_{2}, x_{3}, x_{1}\right), f\left(x_{3}, x_{1}, x_{2}\right)\right\}=f\left(x_{1}, x_{2}, x_{3}\right)$ is an iterative equation of degree three and order three.

The preceding classification of equations on the basis of order and degree necessarily involves the finiteness of the number of variables. It appears possible, however, to define at least formally extensions of categories of functional equations previously defined by the author to equations of infinite degree. In particular, one can give formal definitions of transitive and quasi-transitive equations of infinite degree and order $k$ where $k$ is a positive integer. For example, $\phi\left\{u_{1}, u_{2}, \cdots, u_{n}, \cdots\right\}=\psi\left(x_{1}, x_{2}, \cdots, x_{n}, \cdots\right)$ where $u_{i}=\lambda_{i} f\left(x_{i}, t_{1}, t_{2}, \cdots, t_{n}, \cdots\right)(i=1,2, \cdots)$ is of infinite degree and order 1 . The domain of validity of any of these formally defined equations is, of course, a matter of special investigation.

* Cf. Bulletin, vol. 23 (1916), pp. 76-78.

$\dagger$ Cf. Bulletin, previous abstracts. 
On the basis of the properties of an abstract field, one defines directly an abstract relation having $n+1$ elements as arguments $(n=1,2, \cdots)$ and whose functional interpretation is the equation $f\left\{u_{1}, u_{2}, \cdots, u_{n+1}\right\}=f\left(x_{1}, x_{2}, \cdots, x_{n+1}\right)$, where $u_{i}=f\left\{t_{1}, t_{2}, \cdots, t_{n}, x_{i}\right\}(i=1,2, \cdots, n+1)$. In the latter relation $f\left(x_{1}, x_{2}, \cdots, x_{n+1}\right)=f_{1}\left\{u_{123} \cdots n, u_{2[n+1]}\right\}, n \geqq 2, u_{12}$ $=f_{1}\left(x_{1}, x_{2}\right), u_{123}=f_{1}\left(u_{12}, u_{23}\right), \cdots, u_{123} \cdots n=f_{1}\left(u_{123} \cdots[n-1]\right.$, $\left.u_{2 n}\right)$, and $f_{1}\left\{f_{1}\left(t, x_{2}\right), f_{1}\left(t, x_{1}\right)\right\}=f_{1}\left(x_{1}, x_{2}\right)$.

4. If $A$ is any square matrix, there exists a similar matrix $N$ which is of a well-known canonical form. In a paper in the Annals of Mathematics, September, 1916, Professor Burgess gave a practical method of determining the form of $N$ for any given matrix $A$. In the present paper he gives a practical method for computing the matrix $X$ such that $X^{-1} A X=N$.

The existence of $X$ is well known, but no direct method computing it is to be found in the literature.

5. A résumé of Professor Blumberg's paper is contained in the Proceedings of the National Academy of Sciences, November, 1916. The paper will appear in full in the Annals of Mathematics.

6. Mr. Hathaway groups the residues of a prime $n=6 m$ $+2 \epsilon+3 \quad(\epsilon=1$ or 2$)$, so that $x=a, 1 / a,-1-a$, $-1 /(1+a),-(1+a) / a,-a /(1+a)$, make a group. There are $m$ groups of six residues, one of three residues $(1,-2$, $\left.-\frac{1}{2}\right)$, and $\epsilon$ of two residues, $(0,-1)$ and, when $\epsilon=2,(\omega$, $\left.\omega^{2}=-1-\omega\right)$.

The function $y=\left(1+x+x^{2}\right)^{3} /\left(x+x^{2}\right)^{2}$ is invariant for a group, and designating the values for the $m+1$ groups by $y_{r}, r=0,1, \cdots, m$, in which $y_{m}=27 / 4$, the invariant of the group of three, the numbers of quadratic and non-quadratic residues $y_{r}$ are such that we may assign odd subscripts to the first, and even subscripts to the second.

Taking $\quad \alpha=3(m-r)+\epsilon, \quad \beta=2 r+1, \quad A_{r}=(2 \alpha 3 \beta) / n$ $=\Gamma(\alpha+\beta) / \Gamma(\alpha+1) \Gamma(\beta+1)$, to define the functions, $\varphi(y)$ $=\Sigma A_{r} y^{m-r}, F(y)=\Sigma \beta A_{r} y^{m-r}, F_{1}(y)=\Sigma \beta A_{r} y^{m-r} /(\beta+1)$, the congruences $F(y) \equiv 0, F_{1}(y) \equiv 0,8 y^{m+1}-3 F_{1}(y) \equiv 0$, mod. $n$, are completely resolvable in $y_{r}$ residues, the first in all except $y_{n}$, the second and third in quadratic and non-quadratic $y$ 's respectively. 
If the sum of three $n$th powers be divisible by $n^{2}$, one of the powers, or the difference of the cubes of two of them, or the resultant of $\varphi(y)=0, F(y)=0$ is divisible by $n$.

7. Professor Van Vleck's paper gives a proof of the following theorem of Haskins (Transactions, April, 1916): Let $f(x)$ and $\phi(x)$ be two functions which are integrable in the sense of Lebesgue over the interval $(a, b)$ and have upper and lower bounds,

then if

$$
h \leqq f(x) \leqq H, \quad h \leqq \phi(x) \leqq H ;
$$

$$
\int_{a}^{b}[f(x)]^{n} d x=\int_{a}^{b}[\phi(x)]^{n} d x \quad(n=1,2,3, \cdots),
$$

the measures of the sets of points $E_{f}(\alpha, \beta)$ and $E_{\phi}(\alpha, \beta)$ for which $f(x)$ and $\phi(x)$ lie between $\alpha$ and $\beta$ inclusive will be equal for all pairs of values $(\alpha, \beta)$ included in the function range $(h, H)$. Professor Van Vleck reduces this theorem to a special case of the problem of moments of Stieltjes and then establishes it by this author's results.

8. A class of curves in space of $n+1$ dimensions may be defined by the conditions that each curve of the class must satisfy a set of differential equations of the form

$$
\varphi_{a}\left(x ; y_{1}, \cdots, y_{n} ; y_{1}{ }^{\prime}, \cdots, y_{n}{ }^{\prime}\right)=0 \quad(\alpha=1,2, \cdots, m<n)
$$

and pass through two fixed points. The problem of Lagrange is to characterize a curve of the class which makes an integral

$$
\int f\left(x ; y_{1}, \cdots, y_{n} ; y_{1}{ }^{\prime}, \cdots, y_{n}{ }^{\prime}\right) d x
$$

a maximum or minimum. It is customary to assume that along the particular curve in question the determinant of the first $m$ rows of the matrix $\left\|\partial \varphi_{a} / \partial y_{k}{ }^{\prime}\right\|(\alpha=1, \cdots, m ; k=1$, $\cdots, n)$ is everywhere different from zero. This is an unfortunately unsymmetric assumption, and it is the purpose of the paper of Professor Bliss to show that the details of the theory can be carried through if the matrix is only assumed to be of rank $m$ at each point of the minimizing or maximizing curve. The device used is the addition of $n-m$ variables $z_{r}$ $(r=m+1, \cdots, n)$, and $n-m$ differential equations

$$
\varphi_{r}\left(x ; y_{1}, \cdots, y_{n} ; y_{1}{ }^{\prime}, \cdots, y_{n}{ }^{\prime}\right)=z_{r} \quad(r=m+1, \cdots, n),
$$


such that the determinant $\left|\partial \varphi_{i} / \partial y_{k}{ }^{\prime}\right|(i, k=1, \cdots, n)$ is everywhere different from zero on the curve to be studied. The results are of course independent of the auxiliary equations and variables introduced. A similar method is applicable to the problem of Mayer.

9. Professor Sisam's paper discusses the relation between the order of the curves of a system of algebraic curves on an algebraic surface, the number of variable points common to two generic curves of the system, and the order of the surface. It determines a new sufficient condition that such a system of curves constitute a pencil.

11. In general, the proofs of the existence of the solutions of differential equations establish their existence only in limited domains. In this paper Professor Moulton gives a practical method, related closely to that known as Picard's method, for constructing the solutions so long as the independent and dependent variables remain in the region for which the differential equations have certain specified properties. By suitable specialization, the process reduces to the Cauchy-Lipschitz method. The validity of the method of mechanical quadratures employed by astronomers and physicists is established. Finally, corresponding results are established for a certain type of an infinite system of differential equations in infinitely many variables.

12. In the present paper Professors Bauer and Slobin study a system of three equations of the form

$$
\varphi_{1}(x, y)=0, \quad \varphi_{2}(x, y)=0, \quad T(x, y)=0,
$$

where the $\varphi_{i}$ 's are polynomials in $x$ and $y$ with algebraic coefficients, and where $T(x, y)=0$ denotes an equation which, in general, is not satisfied if $x$ and $y$ are both algebraic numbers. As illustrations of equations of this type, may be mentioned

$$
\begin{gathered}
x-e^{y}=0, y-\sin x=0, \varphi_{1}(x, y)+\varphi_{2}(x, y) \tan \varphi_{3}(x, y)=0, \\
\varphi_{1}(x, y) e^{\phi_{2}(x, y)}+\varphi_{3}(x, y)=0 .
\end{gathered}
$$

The following are some of the theorems considered:

1. The system $A$ cannot have simultaneous solutions (excepting possibly for the pairs of algebraic numbers, if any, 
which satisfy $T(x, y)=0)$. No curves represented by equations of the form $\varphi_{1}(x, y)=0, \varphi_{2}(x, y)=0$ can intersect on the sine curve or the tangent curve excepting at the origin, nor on the exponential curve $x=e^{y}$ excepting in the point $(1,0)$.

2. The system $\varphi_{1}(x, y)+p(t) \varphi_{2}(x, y)=0, \varphi_{3}(x, y)=0$, $\varphi_{4}(x, y)=0$ can have no simultaneous solutions except those, if any, which simultaneously satisfy $\varphi_{1}=0, \varphi_{2}=0$, $\varphi_{3}=0, \varphi_{4}=0$, where $p(t)$ is a polynomial in $t$, a transcendental number.

3. All the singularities of the curves represented by $\psi(x, y)$ $\equiv \varphi_{1}(x, y)+t \varphi_{2}(x, y)=0$ which require $\partial \psi / \partial x=0$ and $\partial \psi / \partial y=0$, lie upon $\varphi_{1}(x, y)=0$ and $\varphi_{2}(x, y)=0$ if there are any at all.

4. All singularities of $\varphi_{0}(x, y)+p_{1}(t) \varphi_{1}(x, y)+\cdots$ $+p_{n}(t) \varphi_{n}(x, y)=0$ must lie upon each of the curves represented by the equations $\varphi=0, \varphi_{1}=0, \cdots, \varphi_{n}=0$ where $p_{i}(t),(i=1, \cdots, n)$, are polynomials in $t$, a transcendental number, the coefficients of the polynomials being algebraic numbers.

13. In this paper, Professor Bennett examines by elementary methods the form of a closed algebraic correspondence upon an algebraic curve or Riemann surface. As a result of certain elementary properties of involutions, and the group properties of closed algebraic and finite systems of points, the relations between closed correspondences and variable inscribed plane configurations are described. A systematic method of securing fundamentally different generalizations of the closure problems arising in connection with Poncelet polygons is obtained.

ARNold Dresden, Secretary of the Section.

\section{THE TWENTY-THIRD ANNUAL MEETING OF THE AMERICAN MATHEMATICAL SOCIETY.}

The twenty-third annual meeting of the Society, which was held in New York City on Wednesday and Thursday, December $27-28,1916$, was in several respects an exceptional occasion. It took place in the midst of the convocation week series of meetings of the American association for the advance- 